\title{
Unitary Implementation of Automorphism Groups on von Neumann Algebras *
}

\author{
HERBERT HALPERN \\ University of Cincinnati, Cincinnati, Ohio USA
}

Received October 2, 1971

\begin{abstract}
A necessary and sufficient continuity condition is obtained in order that a topological group of automorphisms of a semi-finite von Neumann algebra in standard form is unitarily implemented. The methods used are extended to the study of unitary implementation for a general von Neumann algebra of those automorphism groups that commute with the one-parameter modular automorphism group.
\end{abstract}

\section{Introduction}

The Hilbert space $H$ of a semi-finite von Neumann algebra $\mathfrak{A}$ in standard form can be viewed as the completion of a certain two-sided ideal $\mathrm{m}^{1 / 2}$ of $\mathfrak{A}$. It is then not surprising that an automorphism (i.e. a *-automorphism) $\gamma$ of $\mathfrak{A}$ will be implemented by a unitary operator $U$ on $H$ in the sense that $\gamma(A)=U A U^{-1}$ for every $A$ in $\mathfrak{A}$. If $\Gamma$ is a group of automorphisms of $\mathfrak{A}$, one might conjecture that there is a homomorphism $U$ of $\Gamma$ into the group of unitary operators on $H$ so that the homomorphism $\gamma \rightarrow U_{\gamma}$ implements the action of $\Gamma$ in the sense that $\gamma(A)$ $=U_{\gamma} A U_{\gamma}^{-1}$ for every $A \in \mathfrak{A}$ and $\gamma \in \Gamma$. If $\Gamma$ is a topological group and if the action of $\Gamma$ is continuous in the sense that for every fixed $A \in \mathfrak{A} \gamma \rightarrow \gamma_{0}$ in $\Gamma$ implies $\gamma(A) \rightarrow \gamma_{0}(A)$ in the weak operator topology of $\mathfrak{U}$, then one wishes $U$ to be continuous in the sense that $U_{\gamma} \rightarrow U_{\gamma_{0}}$ in the strong (equivalently, weak) operator topology of $H$ whenever $\gamma \rightarrow \gamma_{0}$.

We shall show that every continuous automorphism group of semifinite von Neumann algebra in standard form on the Hilbert space $H$ is implemented by a (continuous) unitary representation on $H$, provided a certain joint continuity condition holds. We show that every continuous locally compact automorphism group automatically has this joint continuity property by showing that a continuous locally compact automorphism group of an arbitrary von Neumann algebra is unitarily implemented on some Hilbert space (depending presumably on the group) on which the algebra is faithfully represented as a von Neumann algebra.

* This research was partially supported by the National Science Foundation. 
The methods of proof are found in the literature. The main contribution here is expressing the objects involved in a form that these methods can be applied. This involves the factorization of functionals through the center of the algebra and then calculating with the resulting measurable functions on the spectrum of the center. Once we make this factorization, we use the method of Guichardet and Kastler [13, proposition 5], to prove unitary implementation. Complications still arise here due to the absence of the $L^{1}$ structure of [13] because of the more general hypothesis. The method of [13] also is the same of R. Busby and H. Smith $[5 ; \S 5]$, even though those authors assumed that $\mathrm{m}^{1 / 2}$ is invariant under $\Gamma$ and also considered no topological questions.

Our main theorem is phrased in a more general way than is need for the semi-finite case. This is done to include automorphism groups that are "liés" in the terminology of [13] with respect to a faithful normal functional given by a cyclic vector on a von Neumann algebra. We show what automorphism groups have this property in terms of their relation to the modular automorphism group of the functional [23, cf. $21, \S 13]$ and then show these groups are unitarily implemented. Aside from continuity we feel that here the proof of [13] applies directly; however, the hypotheses on the algebra and the group in [13] are extremely restrictive and so we do a very minimal amount of extra work in our main Theorem 8 so that there should be no question concerning the validity of the implementation.

As corollaries we obtain results that generalize the results of Aarnes [1] and remove the separability condition on the group from [14] in the semi-finite case.

\section{Continuity}

Let $\mathfrak{A}$ be a von Neumann algebra and let $\mathfrak{A}_{*}$ be the predual of $\mathfrak{A}$, i.e. the space of $\sigma$-weakly continuous functionals on $\mathfrak{A}$. Let $\Gamma$ be a topological group of automorphisms of $\mathfrak{A}$; the group $\Gamma$ is said to be a continuous automorphism group of $\mathfrak{A}$ if, for every $A \in \mathfrak{A}$ and $\phi \in \mathfrak{A}_{*}$, the function $\gamma \rightarrow \phi(\gamma(A))$ is continuous on $\Gamma$. The group $\Gamma$ is said to be jointly continuous at a point $(\gamma, A)$ of the cartesian product of $\Gamma$ and a subset $\mathfrak{B}$ of $\mathfrak{A}$ if, for every $\phi \in \mathfrak{A}_{*}$, the function $\left(\gamma^{\prime}, A^{\prime}\right) \rightarrow \phi\left(\gamma^{\prime}\left(A^{\prime}\right)\right)$ is continuous at $(\gamma, A)$ on $\Gamma \times \mathfrak{B}$, where $\mathfrak{B}$ is taken with the relativized $\sigma$-weak topology. If $\Gamma$ is jointly continuous at $(\varepsilon, 0)$ on $\Gamma \times \mathfrak{B}$, then $\Gamma$ is said to be jointly continuous at the origin on $\Gamma \times \mathfrak{B}$. Here $\varepsilon$ is the neutral element of $\Gamma$. Aarnes studied joint continuity at the origin on the cartesian product of $\Gamma$ and the positive elements in the unit sphere of $\mathfrak{A}$.

A topological group $\Gamma$ of automorphisms of a von Neumann algebra $\mathfrak{Q}$ on a Hilbert space $H$ is said to be unitarily implemented on $H$ if there is 
a unitary representation $U$ of $\Gamma$ on $H$ such that $\gamma(A)=U_{\gamma} A U_{\gamma}^{-1}$ for every $A \in \mathfrak{A}$ and $\gamma \in \Gamma$. The group $\Gamma$ is said to be unitarily implemented if there is a faithful representation $\pi$ of the von Neumann algebra $\mathfrak{A}$ onto a von Neumann algebra $\mathfrak{B}$ on a Hilbert space $K$ and a unitary representation $U$ of $\Gamma$ on $K$ such that $\pi(\gamma(A))=U_{\gamma} \pi(A) U_{\gamma}^{-1}$ for all $A \in \mathfrak{Q}$ and $\gamma \in \Gamma$. (Such a representation $\pi$ is called covariant.) In the sequel all unitary representations of a topological group are assumed to be strongly continuous (i.e. continuous with regard to the strong operator topology). An algebraic homomorphism of a group into the unitary operators on a Hilbert will simply be called a homomorphism.

We need the following lemma of Guichardet and Kastler [13; proposition 3].

Lemma. Let $\mathfrak{A}$ be a von Neumann algebra on the Hilbert space H, and let $\Gamma$ be a topological automorphism group of $\mathfrak{A}$ that is unitarily implemented on $H$. Then, for every $\phi$ in the predual $\mathfrak{A}_{*}$ of $\mathfrak{A}$, the function $\gamma \rightarrow \phi \cdot \gamma$ is continuous map of $\Gamma$ into $\mathfrak{A}_{*}$ taken with its norm topology.

The first proposition indicates the kind of hypothesis we must examine to find a sufficient condition for unitary implementation.

Proposition 1. A unitarily implemented topological group $\Gamma$ of automorphisms of a von Neumann algebra $\mathfrak{A}$ is jointly continuous on the cartesian product of $\Gamma$ and any bounded subset of $\mathfrak{A}$.

Proof. For every $\phi \in \mathfrak{A}_{*}$, the function $\gamma \rightarrow \phi \cdot \gamma$ is continuous function of $\Gamma$ into $\mathfrak{A}_{*}$ with its uniform topology by the lemma of Guichardet and Kastler. Let $\mathfrak{B}$ be a bounded subset of $\mathfrak{I}$, let $A \in \mathfrak{B}$, and let $\gamma \in \Gamma$. Given $\varrho>0$, there is a neighborhood $N(\gamma)$ of $\gamma$ such that

$$
\left\|\phi \cdot \gamma^{\prime}-\phi \cdot \gamma\right\| \leqq \varrho(1+\operatorname{lub}\{\|A\| \mid A \in \mathfrak{B}\})^{-1}
$$

whenever $\gamma^{\prime} \in N(\gamma)$. If $\left|\phi \cdot \gamma\left(A^{\prime}-A\right)\right| \leqq \varrho$, then

$$
\left|\phi \cdot \gamma^{\prime}\left(A^{\prime}\right)-\phi \cdot \gamma(A)\right| \leqq\left|\left(\phi \cdot \gamma^{\prime}-\phi \cdot \gamma\right)\left(A^{\prime}\right)\right|+\left|\phi \cdot \gamma\left(A^{\prime}-A\right)\right| \leqq 2 \varrho
$$

Q.E.D.

We now show that in one case at least a continuous automorphism group on a von Neumann algebra is automatically jointly continuous. In fact, we prove that a continuous locally compact automorphism group is unitarily implemented. For this, we use a construction of Henle [14]. Henle used countable structures and so was able to base the construction on the measurable fields of [9; Chapter II]. Since it is wellknown that certain arguments fail without countability, we sketch a construction in the hope that a certain amount of repetition will be acceptable in the interest of clarity. The construction is more closely related to ideas in Fell [12] than in [9]. 
We need the following result of Aarnes.

Lemma [1, Corollary, p. 333]. If $\Gamma$ is a continuous locally compact automorphism group of a von Neumann algebra, then $\Gamma$ is jointly continuous at the origin on the cartesian product of $\Gamma$ and the positive unit sphere of the algebra.

Proposition 2. Every continuous locally compact automorphism group of a von Neumann algebra is unitarily implemented.

Proof. Let $\Gamma$ be a continuous locally compact automorphism group of the von Neumann algebra $\mathfrak{A}$ on the Hilbert space $H$. Let $\mu$ be right invariant Haar measure on $\Gamma$. Let $K(\Gamma)$ be the algebra of continuous complex-valued functions with compact support on $\Gamma$ and let $K$ be the linear subspace of all functions of $\Gamma$ into $H$ generated by functions $f \cdot x$ defined by $(f \cdot x)(\gamma)=f(\gamma) x$, where $f \in K(\Gamma)$ and $x \in H$. A positive hermitian form can be defined on $K$ by setting

$$
\left\langle\Sigma f_{i} \cdot x_{i}, \Sigma g_{i} \cdot y_{i}\right\rangle=\int_{\Gamma} \sum_{i, j} f_{i}(\gamma) g_{j}(\gamma)^{-}\left(x_{i}, y_{j}\right) d \mu,
$$

for $f_{i}, g_{i}$ in $K(\Gamma)$ and $x_{i}, y_{i}$ in $H$. The set

$$
K_{0}=\{x \in K \mid\langle x, x\rangle=0\}
$$

is a subspace of $K$ and is equal to $K_{0}=\{x \in K \mid\langle x, y\rangle=0$, for all $y \in K\}$. The hermitian form (1) may be transferred to an inner product

$$
\left\langle x+K_{0}, y+K_{0}\right\rangle=\langle x, y\rangle
$$

on the factor space $K-K_{0}=H_{0}$. The completion of the pre-Hilbert space $H_{0}$ is denoted by $L^{2}(\Gamma, H)$. Notice that we have written the inner product in $L^{2}(\Gamma, H)$ as $\langle\cdot, \cdot\rangle$ to distinguish it from the inner product of $H$.

For every $A \in \mathfrak{U}, f_{1}, \ldots, f_{n}, g_{1}, \ldots, g_{m}$ in $K(\Gamma)$ and $x_{1}, \ldots, x_{n}, y_{1}, \ldots, y_{m}$ in $H$, let

$$
\pi\left(A, \Sigma f_{i} \cdot x_{i}+K_{0}, \Sigma g_{i} y_{i}+K_{0}\right)=\int \sum_{i, j} f_{i}(\gamma) g_{j}(\gamma)^{-}\left(\gamma(A) x_{i}, y_{j}\right) d \mu .
$$

Then for every $A$ in $\mathfrak{A}$ there is a unique bounded linear operator $\pi(A)$ on $L^{2}(\Gamma, H)$ such that

$$
\langle\pi(A) x, y\rangle=\pi(A, x, y),
$$

for every $x, y$ in $H_{0}$. The map $\pi$ is a faithful representation of $\mathfrak{A}$ on $L^{2}(\Gamma, H)$. This completes the outline of the construction.

Now we show that $\pi$ is $\sigma$-weakly continuous. If $\left\{E_{i}\right\}$ is a monotonely increasing net of projections in $\mathfrak{U}$ with least upper bound $E$, then it is sufficient to show that $\operatorname{lub}\left\langle\pi\left(E_{i}\right) y, y\right\rangle=\langle\pi(E) y, y\rangle$ for every $y \in L^{2}(\Gamma, H)$ of the form $y=f \cdot x+K_{0}$, where $f$ is a continuous function of compact support $\Gamma_{0}$ on $\Gamma$ and $x \in H$. Now given $\varrho>0$, by the lemma of Aarnes, we 
may find a neighborhood $N$ of the neutral element $\varepsilon$ of $\Gamma$ and a $\sigma$-weak neighborhood $N^{\prime}$ of 0 in $\mathfrak{A}$ such that $(\gamma(A) x, x)<\varrho$ whenever $\gamma \in N$ and $A \in N^{\prime} \cap \mathfrak{A}^{+}$with $\|A\| \leqq 1$. Let $\gamma_{1}, \ldots, \gamma_{n}$ be elements of $\Gamma$ such that $\left\{N \gamma_{i}\right\}$ covers $\Gamma_{0}$, and let $i_{0}$ be an index such that $\gamma_{j}\left(E-E_{i}\right) \in N^{\prime}$ for $i \geqq i_{0}$ and $j=1, \ldots, n$. Then we have that

$$
\left(\gamma\left(E-E_{i}\right) x, x\right)<\varrho
$$

for all $i \geqq i_{0}$ and all $\gamma \in \Gamma_{0}$. This means that

$$
\langle\pi(E) y, y\rangle-\left\langle\pi\left(E_{i}\right) y, y\right\rangle \leqq \operatorname{lub}\left\{|f(\gamma)|^{2}: \gamma \in \Gamma\right\} \mu\left(\Gamma_{0}\right) \varrho,
$$

for every $i \geqq i_{0}$. Thus lub $\left\langle\pi\left(E_{i}\right) y, y\right\rangle=\langle\pi(E) y, y\rangle$.

Now, we argue as in Henle [14]. For every $\gamma \in \Gamma$ and $f \in K(\Gamma)$, let $f^{\gamma} \in K(\Gamma)$ be given by $f^{\gamma}(\delta)=f(\delta \gamma)$. Then given $f_{1}, \ldots, f_{n}, g_{1}, \ldots, g_{m}$ in $K(\Gamma)$ and $x_{1}, \ldots, x_{n}, y_{1}, \ldots, y_{m}$ in $H$, we have that

$$
\left\langle\Sigma f_{i}^{\gamma} \cdot x_{i}+K_{0}, \Sigma g_{i}^{\gamma} \cdot y_{i}+K_{0}\right\rangle=\left\langle\Sigma f_{i} \cdot x_{i}+K_{0}, \sum g_{i} \cdot y_{i}+K_{0}\right\rangle .
$$

Hence, for each $\gamma \in \Gamma$, there is a unique unitary operator $U_{\gamma}$ on $L^{2}(\Gamma, H)$ satisfying

$$
U_{\gamma}\left(\Sigma f_{i} \cdot x_{i}+K_{0}\right)=\Sigma f_{i}^{\gamma} \cdot x_{i}+K_{0}
$$

for every $f_{1}, \ldots, f_{n}$ in $K(\Gamma)$ and $x_{1}, \ldots, x_{n}$ in $H$. Furthermore the map $\gamma \rightarrow U_{\gamma}$ is a unitary representation of $\Gamma$ on $L^{2}(\Gamma, H)$. However, an easy calculation also yields

$$
U_{\gamma} \pi(A) U_{\gamma}^{-1}=\pi(\gamma(A)),
$$

for every $A \in \mathfrak{A}$ and $\gamma \in \Gamma$. Q.E.D.

The next corollary extends the lemma of Aarnes.

Corollary 3. Every continuous locally compact topological group of automorphisms of a von Neumann algebra is jointly continuous on the Cartesian product of the group and any bounded subset of the algebra.

Proof. Let the notation be the same as Proposition 2. By Proposition 1 the map $(\gamma, A) \rightarrow U_{\gamma} A U_{\gamma}^{-1}$ is jointly continuous at $\left(\gamma_{0}, \pi\left(A_{0}\right)\right)$ on the cartesian product of $\Gamma$ and the image $\pi(\mathfrak{B})$ of a bounded subset $\mathfrak{B}$ of $\mathfrak{A}$. Since $\pi$ and $\pi^{-1}$ are $\sigma$-weakly continuous, the map

$$
(\gamma, A) \rightarrow \pi^{-1}\left(U_{\gamma} \pi(A) U_{\gamma}^{-1}\right)=\gamma(A)
$$

is jointly continuous at $\left(\gamma_{0}, A_{0}\right)$ on $\Gamma \times \mathfrak{B}$. Q.E.D.

We can obtain a new proof of a result of Aarnes [2; Proposition 6.1].

Corollary 4. Let $\mathfrak{A}$ be a von Neumann algebra, let $\mathfrak{A}_{*}$ be the predual of $A$, and let $\Gamma$ a continuous locally compact automorphism group of $\mathfrak{A}$. Then, for every $\phi \in \mathfrak{U}_{*}$, the map $\gamma \rightarrow \phi \cdot \gamma$ is continuous where $\mathfrak{A}_{*}$ is taken with its norm topology. 
Proof. Proposition 2 and Lemma of Guichardet and Kastler.

It is now possible to improve some continuity conditions that are found in the literature. Let $\mathfrak{B}$ be a $C^{*}$-algebra with identity on a Hilbert space $H$. Let $\mathfrak{A}$ be the von Neumann algebra generated by $\mathfrak{B}$ on $H$ and let $\mathfrak{A}_{*}$ be the predual of $\mathfrak{A}$. Let $\Gamma$ be a locally compact group of automorphisms of $\mathfrak{B}$. For each $\gamma \in \Gamma$ and each $\phi \in \mathfrak{U}_{*}$, there is one and only one functional $\phi_{\gamma} \in \mathfrak{U}_{*}$ satisfying the relation $\phi_{\gamma}(A)=\phi(\gamma(A))$ for every $A \in \mathfrak{B}$ (cf. [2; p. 334]). Aarnes [2; Theorem] showed the following statements are equivalent:

(1) each $\gamma \in \Gamma$ can be uniquely extended to an automorphism $\gamma^{\prime}$ of $\mathfrak{A}$ such that $\left\{\gamma^{\prime} \mid \gamma \in \Gamma\right\}$ is a continuous automorphism group of $\mathfrak{A}$; and (2) $\gamma \rightarrow \phi_{\gamma}$ is a continuous map of $\Gamma$ into $\mathfrak{U}_{*}$ with its $\sigma\left(\mathfrak{U}_{*}, \mathfrak{U}\right)$ topology. By Corollary 4 (2) may be replaced by the following statement

$\left(2^{\prime}\right) \gamma \rightarrow \phi_{\gamma}$ is a continuous map of $\Gamma$ into $\mathfrak{U}_{*}$ with its uniform topology.

If the Hilbert space $H$ is separable and if the topology of $\Gamma$ has a countable base, then Kallman [17] proved that (1) is satisfied whenever each $\gamma$ can be extended to an automorphism $\gamma^{\prime}$ of $\mathfrak{U}$ and $\gamma \rightarrow \phi_{\gamma}$ is continuous in the $\sigma\left(\mathfrak{U}_{*}, \mathfrak{B}\right)$-topology of $\mathfrak{A}_{*}$.

Now as a special case of Theorem III.1 of Borchers [3], the identity representation of $\mathfrak{B}$ on $H$ is covariant extendible (i.e., is unitarily equivalent to a subrepresentation of a covariant representation) provided (i) that $\Gamma$ is strongly continuous on $\mathfrak{B}$ in the sense that, for every $B$ in $\mathfrak{B}$, the map $\gamma \rightarrow \gamma(B)$ is continuous from $\Gamma$ into $\mathfrak{B}$ with its norm topology and (ii) that, for every vector state $\omega$ of $H$, the map $\gamma \rightarrow \omega \cdot \gamma$ is continuous from $\Gamma$ into the dual $\mathfrak{B}^{*}$ of $\mathfrak{B}$ with its norm topology. Due to the Kaplansky density theorem [9; I, $\S 3$, Theorem 3], the condition (ii) implies (1) via $\left(2^{\prime}\right)$. By Proposition 2, there is a faithful normal representation $\pi$ of $\mathfrak{A}$ on a Hilbert space $H_{\pi}$ such that $\left\{\gamma^{\prime} \mid \gamma \in \Gamma\right\}$ is unitarily implemented on $H_{\pi}$. This means that the identity representation of $\mathfrak{B}$ on $H$ is quasi-equivalent $[8 ; \S 5]$ to a covariant representation of $\mathfrak{B}$ (i.e., the identity representation is quasi-covariant, in the terminology of [3]). Since every multiple of a covariant representation is covariant, the identity representation is covariant extendible (cf. $[8 ; 5.3 .1]$ ). Hence, we see that condition (ii) is necessary and sufficient for the identity representation to be quasicovariant. In general, let $\pi$ be a representation of the $C^{*}$-algebra $\mathfrak{B}$ on the Hilbert space $K$. Then we see from Proposition 2 that $\pi$ is quasi-covariant if and only if (ii) $\gamma \rightarrow \omega \cdot \pi \cdot \gamma$ is a continuous map from $\Gamma$ to $\mathfrak{B}^{*}$ with its norm topology for every vector state $\omega$ on $K$, and (iii) the kernel of $\pi$ is invariant under $\Gamma$. Borchers [3; III.1] showed that, if $\Gamma$ is strongly continuous, the condition (ii) is necessary and sufficient for a representation to be covariant extendible. The strong continuity is hypothesized so that the covariant algebra [10] can be employed. 


\section{Unitary Implementation}

Let 3 be a commutative von Neumann algebra. There is a locally compact space $Z$ and a Radon measure $v$ on $Z$ so that 3 with its $\sigma$-weak topology is identified (i.e. isomorphic) with the algebra $L^{\infty}=L_{C}^{\infty}(Z, v)$ of all essentially bounded complex-valued measurable functions on $Z$ with its $\sigma$-weak topology, i.e. with the topology induced on $L^{\infty}$ by $L_{C}^{1}(Z, v)$. Let $\hat{3}^{+}$be the set of all positive, finite or infinite valued, measurable functions on $Z$. Functions in $\hat{3}^{+}$which are equal locally almost everywhere are identified with each other in the same way the functions of $L^{\infty}$ are identified. The set $3^{+}$is embedded in $\hat{3}^{+}$in a natural way. Since each majorized monotonely increasing net in $3^{+}$has a least upper bound in $3^{+}$, each monotonely increasing net in $\hat{3}^{+}$has a least upper bound in $\hat{3}^{+}$so that the least upper bound in $\hat{3}^{+}$of a majorized monotonely increasing net in $3^{+}$is also in $3^{+}$. Notice that the least upper bounds are in general not pointwise least upper bounds. For every $S$ in $\hat{3}^{+}$, let $\omega(S)$ denote the essential upper integral of $S$. The function $\omega$ of $\hat{3}^{+}$is a normal semi-finite faithful trace of $3^{+}$whose restriction to $3^{+}$is a normal semi-finite faithful trace of 3 [6 and 9; III, $\S 4]$. Without further mention we shall always assume some choice of $Z$ and $v$ has been made for every abelian von Neumann algebra.

Any element $Q$ in $\hat{\mathbf{3}}^{+}$is actually a class of functions. Any two elements of the class differ from each other on a set which is locally of measure 0 i.e. a set whose intersection with each compact set is of measure zero. As is customary, we consider algebraic relations for the elements of $\hat{\mathbf{3}}^{+}$as though they were actual functions with the modifier locally almost everywhere adjoined. This means that two different choices of representing measurable functions for the various classes involved in the algebraic relations give two different resulting measurable functions which lie in the same class in $\hat{3}^{+}$i.e. which are equal locally almost everywhere. In the sequel, we pass freely from classes to representations of the classes. Underlying this, however, is the assumption that verification has been made that such passage is legitimate.

Let $Q$ be an element of $\hat{3}^{+}$such that $0<Q(\zeta)<+\infty$ locally almost everywhere on $Z$. For every $n=1,2, \ldots$, the characteristic function $E_{n}$ of the measurable set $\left\{\zeta \in Z \mid n^{-1} \leqq Q(\zeta) \leqq n\right\}$ is a projection in $L^{\infty}$. The sequence $\left\{E_{n}\right\}$ of projections is clearly monotonely increasing and bounded above by the constant function 1 . We show that lub $E_{n}=E$ is equal to 1 . Indeed, let $P$ be the characteristic function of any compact set $X$ in $Z$. By the definition of locally almost everywhere, we have that $\{\zeta \in X \mid Q(\zeta)=0$ or $Q(\zeta)=+\infty\}$ is a set of measure zero. Hence, the set $X-X \cap\left(\cup\left\{\zeta \in Z \mid n^{-1} \leqq Q(\zeta) \leqq n\right\}\right.$ has measure zero. Thus $E P=P$ 
locally almost everywhere. This proves that the function $E$ is equal to 1 locally almost everywhere.

Now let $\gamma$ be an automorphism of 3 . The element $E_{n} Q$ determines a class of essentially bounded measurable functions and so $E_{n} Q \in 3^{+}$. The sequence $\left\{\gamma\left(E_{n} Q\right)\right\}$ is monotonely increasing in $3^{+}$since $\left\{E_{n} Q\right\}$ is monotonely increasing in $3^{+}$. We denote the least upper bound of $\left\{\gamma\left(E_{n} Q\right)\right\}$ in $\hat{3}^{+}$by $\gamma(Q)$. lemma.

We make some preparations for the Theorem 8 in the following

Lemma 5. Let 3 be an abelian von Neumann algebra and let $\gamma$ be an automorphism of 3. Let $Q$ and $R$ be elements of $\hat{3}^{+}$such that $0<Q(\zeta)<+\infty$ and $0<R(\zeta)<+\infty$ locally almost everywhere on $Z$. Let $\left\{F_{n}\right\}$ be a monotonely increasing sequence of projections in 3 with least upper bound 1 such that $Q F_{n} \in 3$ for every $n=1,2, \ldots$; then the following statements are true:

(i) $\operatorname{lub} \gamma\left(Q F_{n}\right)$ in $\hat{3}^{+}$is equal to $\gamma(Q)$;

(ii) $0<\gamma(Q)(\zeta)<+\infty$ locally almost everywhere on $Z$;

(iii) $\gamma(Q) \gamma(R)=\gamma(Q R)$; and

(iv) $\gamma\left(Q^{-1}\right)=\gamma(Q)^{-1}$.

Proof. (i) If $\left\{A_{n}\right\}$ is a monotonely increasing sequence of functions in $\hat{3}^{+}$, then the function $A(\zeta)=\operatorname{lub}_{n} A_{n}(\zeta)$ is measurable and hence in $\hat{3}^{+}\left[4 ; \mathrm{IV}, \S 5\right.$, Corollary 1 , Theorem 2]. This means that $\operatorname{lub} A_{n}=A$ locally almost everywhere. Applying this fact to the monotonely increasing sequences $\left\{\gamma\left(Q E_{m} F_{n}\right)\right\}_{m, n}$ and $\left\{\gamma\left(Q E_{m} F_{n}\right)\right\}_{n}$, we get that $\operatorname{lub}_{m, n} \gamma\left(Q E_{m} F_{n}\right) \geqq \operatorname{lub}_{n} \gamma\left(Q E_{m} F_{n}\right)=\gamma\left(Q E_{m}\right)$ locally almost everywhere and then that $\operatorname{lub}_{m, n} \gamma\left(Q E_{m} F_{n}\right) \geqq \operatorname{lub} \gamma\left(Q E_{m}\right)=\gamma(Q)$ locally almost everywhere. Here $E_{m}$ is the characteristic function of the measurable set $\left\{\zeta \in Z \mid m^{-1} \leqq Q(\zeta) \leqq m\right\}$. Since $\gamma\left(Q E_{m} F_{n}\right) \leqq \gamma\left(Q E_{m}\right)$ locally almost everywhere, we obtain the reverse inequality $\gamma(Q) \geqq \operatorname{lub}_{m . n} \gamma\left(Q E_{m} F_{n}\right)$ locally almost everywhere. This means that $\gamma(Q)=\operatorname{lub}_{m, n} \gamma\left(Q E_{m} F_{n}\right)$ locally almost everywhere. Now working with $\left\{\gamma\left(Q F_{n}\right)\right\}$, we obtain lub $\gamma\left(Q F_{n}\right)$ $=\operatorname{lub}_{m, n} \gamma\left(Q E_{m} F_{n}\right)=\gamma(Q)$ locally almost everywhere.

(ii) Since $0<Q(\zeta)<+\infty$ locally almost everywhere, the least upper bound of the projections $\left\{E_{n}\right\}$ defined in (i) is 1 , and so the least upper bound of the monotonely increasing sequence $\left\{\gamma\left(E_{n}\right)\right\}$ is also 1 . Now let $Y$ be a compact subset of $Z$. Then the sets

and

$$
\left\{\zeta \in Y \mid \gamma\left(E_{n}\right)(\zeta)=0 \text { for all } n=1,2, \ldots\right\}
$$

$$
\left\{\zeta \in Y \mid \gamma(Q)(\zeta) \neq \operatorname{lub} \gamma\left(Q E_{n}\right)(\zeta)\right\}
$$


have measure zero as do the complements with respect to $Y$ of the sets

and

$$
\begin{aligned}
& \left\{\zeta \in Y \mid m^{-1} \gamma\left(E_{m}\right)(\zeta) \leqq \gamma\left(Q E_{m}\right)(\zeta) \leqq m \gamma\left(E_{m}\right)(\zeta)\right\}, \\
& \left\{\zeta \in Y \mid \gamma\left(Q E_{n}\right)(\zeta) \gamma\left(E_{m}\right)(\zeta)=\gamma\left(Q E_{m}\right)(\zeta)\right\} \text { for } n \geqq m,
\end{aligned}
$$$$
\left\{\zeta \in Y \mid \gamma\left(Q E_{n}\right)(\zeta) \leqq \gamma\left(Q E_{n+1}\right)(\zeta)\right\} \text { for all } n \text {. }
$$

Thus, there is a subset $Y_{0}$ of $Y$ of measure zero such that $\zeta \in Y-Y_{0}$ implies that $\gamma\left(E_{m}\right)(\zeta)=1$ for some $m$ and that

$$
\begin{aligned}
\gamma(Q)(\zeta) & =\operatorname{lub}_{n} \gamma\left(Q E_{n}\right)(\zeta)=\operatorname{lub}_{n \geqq m} \gamma\left(Q E_{n}\right)(\zeta) \\
& =\operatorname{lub}_{n \geqq m} \gamma\left(Q E_{n}\right)(\zeta) \gamma\left(E_{m}\right)(\zeta)=\gamma\left(Q E_{m}\right)(\zeta)
\end{aligned}
$$

is in the real interval $\left[m^{-1}, m\right]$. So $0<\gamma(Q)(\zeta)<+\infty$ locally almost everywhere.

(iii) Let $G_{n}$ be the characteristic function of the set $\left\{\zeta \in Z \mid n^{-1}\right.$ $\leqq R(\zeta) \leqq n\}$ for every $n=1,2, \ldots$. We have that $\left\{G_{n}\right\}$ is a monotonely increasing sequence of projections of least upper bound 1 and thus that $\left\{E_{m} G_{n}\right\}$ is a monotonely increasing sequence of projections of least upper bound 1. By part (i) we have that $\gamma(Q R)=\operatorname{lub}_{m, n} \gamma\left(Q R E_{m} G_{n}\right)$. But $\gamma(Q R) \geqq \gamma\left(Q E_{m}\right) \gamma\left(R G_{n}\right)$ locally almost everywhere and so $\gamma(Q R)$ $\geqq \gamma(Q) \gamma\left(R G_{n}\right)$ locally almost everywhere and finally $\gamma(Q R) \geqq \gamma(Q) \gamma(R)$ locally almost everywhere. But it is clear that $\gamma\left(Q R E_{m} G_{n}\right) \leqq \gamma(Q) \gamma(R)$ locally almost everywhere. Hence, we have that $\gamma(Q R) \leqq \gamma(Q) \gamma(R)$ locally almost everywhere. Thus we obtain $\gamma(Q) \gamma(R)=\gamma(Q R)$.

(iv) Notice that $0<Q^{-1}(\zeta)<+\infty$ locally almost everywhere and thus $\gamma\left(Q^{-1}\right)$ exists. From (iii) we see that $\gamma\left(Q^{-1}\right) \gamma(Q)=\gamma\left(Q^{-1} Q\right)=\gamma(1)=1$ locally almost everywhere. Thus $\gamma\left(Q^{-1}\right)=\gamma(Q)^{-1}$ in $\hat{3}^{+}$. Q.E.D.

The following definition is purely for convenience. It allows us to treat simultaneously the two cases in which we are interested.

Definition 6. Let $\mathfrak{A}$ be a von Neumann algebra with center 3 . A function $\Phi$ of $\mathfrak{A}^{+}$into $\hat{3}^{+}$will be called an extended normal semi-finite faithful module homomorphism if

(i) $\Phi(A+B)=\Phi(A)+\Phi(B)$ for every $A, B$ in $\mathfrak{U}^{+}$;

(ii) $\Phi(A B)=A \Phi(B)$ for every $A$ in $3^{+}$and $B$ in $\mathfrak{A}^{+}$;

(iii) if $\Phi(A)=0$ for $A$ in $\mathfrak{U}^{+}$, then $A=0$ (faithful);

(iv) the set $\left\{A \in \mathfrak{A}^{+} \mid \omega(\Phi(A))<+\infty\right\}$ is the set of all positive elements of a weakly dense two-sided ideal $\mathfrak{m}$ of $\mathfrak{A}$ (semi-finite);

(v) if $\phi=\omega \cdot \Phi$ on $\mathfrak{A}^{+}$and $\dot{\phi}$ is the unique linear functional on $m$ defined by linearity, then $A \rightarrow \dot{\phi}(A B)$ is $\sigma$-weakly continuous on $\mathfrak{A}$ for every $B \in \mathfrak{m}$ (normal).

If $\Phi$ is an extended normal semi-finite faithful module homomorphism, then the ideal $m$ (iv) will be called the ideal of definition of $\phi=\omega \cdot \Phi$. 
Since $\mathrm{m}$ is dense in $\mathfrak{U}$, there is a monotonely increasing net of projections $\left\{E_{n}\right\}$ in $m$ with least upper bound 1 . Therefore, given any nonzero $A$ in $\mathfrak{I}^{+}$, there is an $E_{n}$ with $A^{1 / 2} E_{n} A^{1 / 2} \neq 0$. This means that every nonzero element of $\mathfrak{A}$ majorizes a nonzero positive element $B$ of $\mathrm{m}$. So the function $\Phi(B)$ is finite locally almost everywhere. Hence, there is a projection $F$ in 3 such that $F \Phi(B)=\Phi(B F)$ is a nonzero element of 3 . This means that $A$ majorizes a nonzero element $B F$ in $m$ such that $\Phi(B F)$ is in 3 .

The set $\mathfrak{m}^{1 / 2}=\left\{A \in \mathfrak{O} \mid A^{*} A \in \mathfrak{m}\right\}$ is a two-sided weakly dense ideal of $\mathfrak{A}[9 ; \mathrm{I}, \S 1$, Proposition 11$]$. The relation

$$
(A, B)=\dot{\phi}\left(B^{*} A\right)
$$

defines an inner product on $\mathrm{m}^{1 / 2}$. The completion of $\mathrm{m}^{1 / 2}$ under this inner product will be denoted by $H_{\phi}$. We notice that $\mathrm{m}$ as well as $\mathrm{m}^{1 / 2}$ is dense in $H_{\phi}$. Indeed, let $A \in\left(\mathrm{m}^{1 / 2}\right)^{+}$and let $\varrho>0$. There is a monotonely increasing sequence $\left\{E_{n}\right\}$ of projections which commute with $A$ and satisfy the relation lub $E_{n}=1$ such that, for each $E_{n}$, there exist orthogonal projections $F_{1}, \ldots, F_{m}$ which also commute with $A$ and positive numbers $\lambda_{1}, \ldots, \lambda_{m}$ which satisfy $(1-\varrho) A E_{n} \leqq \Sigma \lambda_{i} F_{i} \leqq A E_{n}$. This means that $\sum \lambda_{i} F_{i} \in \mathrm{m}$ and

$$
\begin{aligned}
\left\|A-\sum \lambda_{i} F_{i}\right\|_{\phi} & \leqq\left\|A-A E_{n}\right\|_{\phi}+\left\|A E_{n}-\sum \lambda_{i} F_{i}\right\|_{\phi} \\
& \leqq \dot{\phi}\left(\left(1-E_{n}\right) A^{2}\right)^{1 / 2}+\varrho \dot{\phi}\left(A E_{n} A\right)^{1 / 2} \\
& \leqq \dot{\phi}\left(\left(1-E_{n}\right) A^{2}\right)^{1 / 2}+\varrho \dot{\phi}\left(A^{2}\right)^{1 / 2} .
\end{aligned}
$$

Since $\lim _{n} \dot{\phi}\left(\left(1-E_{n}\right) A^{2}\right)=0$ by hypothesis (v), we see that $A$ is in the closure of $m$ in $H_{\phi}$. But each element in $\mathrm{m}^{1 / 2}$ is a linear combination of elements of $\left(\mathrm{m}^{1 / 2}\right)^{+}$. Hence, $\mathrm{m}$ is dense in $\mathrm{m}^{1 / 2}$ or equivalently $\mathrm{m}$ is dense in $H_{\phi}$. For each $A \in \mathfrak{U}$, the map $B \rightarrow A B$ of $\mathrm{m}^{1 / 2}$ into $\mathrm{m}^{1 / 2}$ is a bounded linear operator of $\mathrm{m}^{1 / 2}$ and so the map can be extended uniquely to a bounded linear operator $\pi_{\phi}(A)$ of $H_{\phi}$. The map $\pi_{\phi}$ of $\mathfrak{A}$ into the bounded linear operators of $H_{\phi}$ is a faithful representation of $\mathfrak{A}$ on $H_{\phi}$. It is called the canonical representation of $\mathfrak{A}$ induced by $\phi$. If $\left\{A_{n}\right\}$ is a monotonely increasing net in $\mathfrak{I}^{+}$with least upper bound $A$, then the least upper bound of the monotonely increasing net $\left\{\pi_{\phi}\left(A_{n}\right)\right\}$ is $\pi_{\phi}(A)$. Indeed, we have that $\operatorname{lub}\left(\pi_{\phi}\left(A_{n}\right) B, B\right)=\operatorname{lub} \phi\left(B^{*} A_{n} B\right)=\phi\left(B^{*} A B\right)$ for every $B \in \mathfrak{m}$ since $\lim B^{*} A_{n}=B^{*} A$ ( $\sigma$-weakly). Using the fact that $\mathrm{m}$ is dense in $H_{\phi}$, we conclude that $l u b \pi_{\phi}\left(A_{n}\right)=\pi_{\phi}(A)$.

We now perform a calculation that we shall need.

Lemma 7. Let $\mathfrak{A}$ be a von Neumann algebra with center 3 . Let $\Phi$ be an extended normal faithful semi-finite module homomorphism of $\mathfrak{A}^{+}$into the space $\hat{3}^{+}$which is formed with respect to the normal semi-finite faithful 
trace $\omega$ of 3 . Let $\gamma$ be an automorphism of $\mathfrak{U}$ such that there exists a $Q_{\gamma}$ in $\hat{3}^{+}$with $\omega \cdot Q_{\gamma} \cdot \Phi=\omega \cdot \Phi \cdot \gamma$. Then the following statements are true:

(i) $0<Q_{\gamma}(\zeta)<+\infty$ locally almost everywhere on $Z$; and

(ii) if $Q^{\prime} \in \hat{3}^{+}$and $\omega \cdot\left(Q^{\prime} \Phi\right)=\omega \cdot \Phi \cdot \gamma$, then $Q^{\prime}=Q_{\gamma}$. Suppose $\delta$ is an automorphism of $\mathfrak{U}$ for which there exists a $Q_{\delta}$ in $\hat{3}^{+}$with $\omega \cdot \Phi \cdot \delta$ $=\omega \cdot\left(Q_{\delta} \Phi\right)$; then

(iii) $\omega \cdot \Phi \cdot(\gamma \delta)=\omega \cdot\left(Q_{\delta} \delta^{-1}\left(Q_{\gamma}\right) \Phi\right)$.

Proof. (i) For simplicity let $Q_{\gamma}=Q$. Let $X$ be a measurable subset of a compact set in $Z$, and let $E$ be the projection in 3 which corresponds to the characteristic function of $X$. If $E Q=0$ locally almost everywhere, then $\omega(\Phi(\gamma(E))=0$. This means that $\gamma(E)=0$ since $\omega$ and $\Phi$ are faithful. Hence, the projection $E$ vanishes and $X$ is of measure zero. This means $0<Q(\zeta)$ locally almost everywhere. Now let $X$ be a measurable subset of a compact subset of $Z$ such that $Q(\zeta)=+\infty$ for all $\zeta \in X$. If $X$ is not of measure zero, the characteristic function $E$ is nonzero. For every nonzero $A$ in $\mathfrak{A}$ with $0 \leqq A \leqq E$, we have that $\Phi(A) \in \hat{\mathfrak{Z}}^{+}$is nonzero and majorized by $\Phi(E)=E \Phi(E)$. Hence, the function $Q \Phi(A)$ is equal to $+\infty$ on a set of positive measure. On the other hand, we can find a nonzero $A$ in $\mathfrak{U}$ with $0 \leqq A \leqq E$ such that $\omega \cdot \Phi(\gamma(A))<+\infty$ due to the semi-finiteness of $\Phi$ (cf. discussion following Definition 6). We have obtained a contradiction. Therefore, we have $Q(\zeta)<+\infty$ locally almost everywhere.

(ii) Let $Q^{\prime}$ be in $\hat{3}^{+}$so that $\omega \cdot\left(Q^{\prime} \Phi\right)=\omega \cdot \Phi \cdot \gamma$. By (i), the function $Q^{\prime}$ is finite locally almost everywhere. Let $E$ be a nonzero projection in 3 such that $Q^{\prime} E$ and $Q E$ are in 3 . There is a nonzero $A$ in $\mathrm{m}^{+}$majorized by $E$ such that $\Phi(A)$ is in 3 . This means that

$$
\omega\left(Q^{\prime} E \Phi(A) B\right)=\omega(Q E \Phi(A) B)
$$

for every $B$ in $3^{+}$. Since $\omega$ is a normal semi-finite faithful trace on 3 , we obtain that $Q^{\prime} E \Phi(A)=Q E \Phi(A)$. Hence, we have shown that for every nonzero projection $E$ in 3 such that both $Q^{\prime} E$ and $Q E$ are in 3 , there is a nonzero element $C$ in 3 with $0 \leqq C \leqq E$ such that $Q^{\prime} E C=Q E C$. In view of the fact that $Q^{\prime}$ and $Q$ are finite locally almost everywhere, we find that $Q^{\prime}=Q$.

(iii) Let $F_{n}$ be the characteristic function of the measurable set $\{\zeta \in Z \mid Q(\zeta) \leqq n\}$. For every $A \in \mathfrak{U}^{+}$, we have that

$$
\operatorname{lub} Q_{\delta} \delta^{-1}\left(Q F_{n}\right) \Phi(A)=Q_{\delta} \delta^{-1}(Q) \Phi(A)
$$

in $\hat{3}^{+}($Lemma 5 (i)) and hence that

$$
\operatorname{lub} \omega\left(Q_{\delta} \delta^{-1}\left(Q F_{n}\right) \Phi(A)\right)=\omega\left(Q_{\delta} \delta^{-1}(Q) \Phi(A)\right)
$$


$[4 ; \mathrm{V}, \S 1$, Proposition 1e]. But we have that

$$
\begin{aligned}
\omega\left(Q_{\delta} \delta^{-1}\left(Q F_{n}\right) \Phi(A)\right) & =\omega\left(\Phi\left(\left(Q F_{n}\right) \delta(A)\right)\right)=\omega\left(\left(Q F_{n}\right) \Phi(\delta(A))\right) \\
& =\omega\left(Q \Phi\left(F_{n} \delta(A)\right)=\omega\left(\Phi\left(\gamma\left(F_{n} \delta(A)\right)\right)\right)\right. \\
& =\omega\left(\gamma\left(F_{n}\right) \Phi(\gamma \delta(A))\right) .
\end{aligned}
$$

Since lub $F_{n}=1$ and so $\operatorname{lub} \gamma\left(F_{n}\right)=1$, we have

$$
\omega\left(Q_{\delta} \delta^{-1}(Q) \Phi(A)\right)=\omega(\Phi(\gamma \delta(A))) \text {. Q.E.D. }
$$

We are now ready to prove our principal theorem. We perform an analysis similar to that of [13; Proposition 5] without recourse to an $L^{1}$-structure or to the fact that the locally compact space $Z$ in [13] can be taken to be the spectrum of 3 ; or equivalently, an analysis similar to that of $[5 ; \S 5]$ without recourse to the invariance of the ideal of definition under the automorphism group.

Theorem 8. Let $\mathfrak{A}$ be a von Neumann algebra with center 3 and let $\Gamma$ be a continuous automorphism group of $\mathfrak{A}$. If $\Gamma$ is not locally compact, then assume that $\Gamma$ is jointly continuous at the origin on the cartesian product of $\Gamma$ and the unit sphere of 3 . Let $\Phi$ be an extended, normal semifinite faithful module homomorphism of $\mathfrak{A}^{+}$into the space $\hat{\mathfrak{3}}^{+}$which is formed with respect to the normal faithful semi-finite trace $\omega$ of 3 . For each $\gamma \in \Gamma$, assume there is a $Q_{\gamma} \in \hat{\mathcal{B}}^{+}$such that $\phi \cdot \gamma=\omega \cdot\left(Q_{\gamma} \Phi\right)$ where $\phi=\omega \cdot \Phi$. Then the automorphism group $\Gamma$ is unitarily implemented on the Hilbert space $H_{\phi}$ associated with the canonical representation induced by $\phi$.

Proof. At the outset we identify each element of $\mathfrak{A}$ with its image under the canonical representation induced by $\phi$.

We have that $0<Q_{\gamma}(\zeta)<+\infty$ locally almost everywhere on $Z$. Hence, the function $Q_{\gamma}^{-1}$ in $\hat{3}^{+}$also satisfies the relation $0<Q_{\gamma}^{-1}(\zeta)<+\infty$ locally almost everywhere on $Z$. The function $R_{\gamma}=\left(Q_{\gamma}^{-1}\right)^{1 / 2}$ in $\hat{\mathbf{3}}^{+}$ satisfies the relation

$$
0<R_{\gamma}(\zeta)<+\infty
$$

locally almost everywhere, and thus $\delta\left(R_{\gamma}\right)$ is defined for every $\delta \in \Gamma$, and is equal to the least upper bound in $\hat{\mathcal{Z}}^{+}$of the sequence $\left\{\delta\left(R_{\gamma} E_{\gamma}^{n}\right)\right\}$, where $E_{\gamma}^{n}$ is the characteristic function of the measurable set $\left\{\zeta \in Z \mid n^{-1}\right.$ $\left.\leqq Q_{\gamma}^{-1}(\zeta) \leqq n\right\}$ or equivalently the set $\left\{\zeta \in Z \mid n^{-1 / 2} \leqq R_{\gamma}(\zeta) \leqq n^{1 / 2}\right\}$ (Lemma 5).

Now we outline the steps of the proof. Let $m$ be the ideal of definition of $\phi$. For every $\gamma \in \Gamma$, we show (I) that the relation $U_{\gamma} A=\lim \gamma\left(R_{\gamma} E_{\gamma}^{n} A\right)$ $\left(A \in \mathrm{m}^{1 / 2}\right)$ uniquely defines a unitary operator $U_{\gamma}$ on $H=H_{\phi}$. Then, using the lemmas we have prepared, we verify (II) that $\gamma \rightarrow U_{\gamma}$ is a homomorphism of the group $\Gamma$ into the group of unitary operators of $H$, 
(III) that $U_{\gamma} A U_{\gamma}^{-1}=\gamma(A)$ for every $A \in \mathfrak{A}$ and $\gamma \in \Gamma$, and finally (IV) that $\gamma \rightarrow U_{\gamma}$ is continuous.

I. We now proceed with the initial step, viz., the definition of $U_{\gamma}$. Let $A \in \mathfrak{m}^{1 / 2}$ and let $\gamma \in \Gamma$. First we show that $\gamma\left(R_{\gamma} E_{\gamma}^{n} A\right) \in \mathfrak{m}^{1 / 2}$. For simplicity, let $R_{\gamma} E_{\gamma}^{n}=R_{\gamma}^{n}$. We have that

$$
\begin{aligned}
\phi\left(\gamma\left(R_{\gamma}^{n} A\right)^{*} \gamma\left(R_{\gamma}^{n} A\right)\right) & =\omega \cdot \Phi\left(\gamma\left(\left(R_{\gamma}^{n}\right)^{2} A^{*} A\right)\right) \\
& =\omega\left(Q_{\gamma}\left(R_{\gamma}^{n}\right)^{2} \Phi\left(A^{*} A\right)\right)=\dot{\phi}\left(E_{\gamma}^{n} A^{*} A\right),
\end{aligned}
$$

for every $n=1,2, \ldots$, since $Q_{\gamma}\left(R_{\gamma}^{n}\right)^{2} \Phi\left(A^{*} A\right)=E_{\gamma}^{n} \Phi\left(A^{*} A\right)$ locally almost everywhere in $\hat{3}^{+}$. Because $A^{*} A \in \mathrm{m}$ and $0 \leqq E_{\gamma}^{n} A^{*} A \leqq A^{*} A$, we see that $\gamma\left(R_{\gamma}^{n} A\right) \in \mathrm{m}^{1 / 2}$.

Now we show that $\left\{\gamma\left(R_{\gamma}^{n} A\right)\right\}$ is a Cauchy sequence in $H$. Indeed, for $n \geqq m$, we have that

$$
\left\|\gamma\left(R_{\gamma}^{n} A\right)-\gamma\left(R_{\gamma}^{m} A\right)\right\|_{\phi}^{2}=\dot{\phi}\left(\left(E_{\gamma}^{n}-E_{\gamma}^{m}\right) A^{*} A\right)
$$

since $Q_{\gamma}\left(R_{\gamma}^{n}-R_{\gamma}^{m}\right)^{2} \Phi\left(A^{*} A\right)=\left(E_{\gamma}^{n}-E_{\gamma}^{m}\right) \Phi\left(A^{*} A\right)$ locally almost everywhere in $\hat{\mathfrak{Z}}^{+}$. The $\sigma$-weak continuity of $B \rightarrow \dot{\phi}\left(B A^{*} A\right)$ on $\mathfrak{A}$ and the $\sigma$-weak convergence of $\left\{E_{\gamma}^{n}\right\}$ to 1 implies that $\left\{\gamma\left(R_{\gamma}^{n} A\right)\right\}$ is Cauchy in $H$. We denote the limit of this Cauchy sequence in $H$ by $U_{\gamma} A$.

It is clear that $U_{\gamma}$ is a linear map of $\mathrm{m}^{1 / 2}$ into $H$. But we also have that

$$
\begin{aligned}
\left(U_{\gamma} A, U_{\gamma} A\right) & =\lim \left(\gamma\left(R_{\gamma}^{n} A\right), \gamma\left(R_{\gamma}^{n} A\right)\right) \\
& =\lim \dot{\phi}\left(E_{\gamma}^{n} A^{*} A\right)=\dot{\phi}\left(A^{*} A\right)=(A, A),
\end{aligned}
$$

for every $A \in \mathrm{m}^{1 / 2}$. This means that $U_{\gamma}$ is an isometry on $\mathrm{m}^{1 / 2}$. Because $\mathrm{m}^{1 / 2}$ is dense in $H$, the map $U_{\gamma}$ has a unique extension to a linear isometry of $H$ into $H$. We also denote this extension by $U_{\gamma}$.

We now complete the proof this initial step by showing that $U_{\gamma}$ is a unitary operator. It is sufficient to show that the range of $U_{\gamma}$ contains an arbitrary $A$ in the dense set $\mathrm{m}^{1 / 2}$ of $H$. Because $\gamma^{-1}\left(R_{\gamma-1}^{n} A\right) \in \mathrm{m}^{1 / 2}$ for every $n=1,2, \ldots$, the element

$$
U_{\gamma}\left(\gamma^{-1}\left(R_{\gamma-1}^{n} A\right)\right)=\lim _{m} \gamma\left(R_{\gamma}^{m}\right) R_{\gamma-1}^{n} A
$$

is in the range of $U_{\gamma}$. However, we have that

$$
\gamma\left(R_{\gamma}^{m}\right) R_{\gamma-1}^{n}=\gamma\left(E_{\gamma}^{m}\right) E_{\gamma-1}^{n}
$$

locally almost everywhere by Lemma 5 (ii), (iii) and (iv) and Lemma 7 (iii). Hence, we have that $\lim _{m} \gamma\left(E_{\gamma}^{m}\right) E_{\gamma-1}^{n} A$ is in the range of $U_{\gamma}$. This means that $E_{\gamma-1}^{n} A$ is in the range of $U_{\gamma}$ as is shown by the relation

$$
\left\|\gamma\left(E_{\gamma}^{m}\right) E_{\gamma-1}^{n} A-E_{\gamma-1}^{n} A\right\|_{\phi}^{2}=\dot{\phi}\left(\left(1-\gamma\left(E_{\gamma}^{m}\right)\right) E_{\gamma-1}^{n} A^{*} A\right)
$$


coupled with the fact that $\left\{\gamma\left(E_{\gamma}^{m}\right)\right\}$ converges $\sigma$-weakly to 1 . Likewise, the relation

$$
\left\|E_{\gamma-1}^{n} A-A\right\|_{\phi}^{2}=\dot{\phi}\left(\left(1-E_{\gamma-1}^{n}\right) A^{*} A\right)
$$

implies that $A$ is in the range of $U_{\gamma}$. Thus, the dense set $\mathrm{m}^{1 / 2}$ is in the range of $U_{\gamma}$. Because $U_{\gamma}$ is an isometry, the range of $U_{\gamma}$ is actually $H$ and so $U_{\gamma}$ is a unitary operator.

II. We now show that $\gamma \rightarrow U_{\gamma}$ is a homomorphism of the group $\Gamma$ into the group of unitary operators of $H$. Let $A \in \mathrm{m}^{1 / 2}$ and let $\gamma$ and $\delta$ be in $\Gamma$. We calculate $U_{\gamma} U_{\delta} A$. We have that

$$
U_{\gamma} U_{\delta} A=\lim _{m} U_{\gamma}\left(\delta\left(R_{\delta}^{m} A\right)\right)=\lim _{m} \lim _{n} \gamma\left(R_{\gamma}^{n} \delta\left(R_{\delta}^{m} A\right)\right)
$$

since $\delta\left(R_{\delta}^{m} A\right) \in \mathrm{m}^{1 / 2}$ (Part I). By Lemmas 5 (iii), (iv), and 7 (ii), (iii) we conclude that

Then we have that

$$
R_{\gamma \delta}=R_{\delta} \delta^{-1}\left(R_{\gamma}\right)
$$

$$
\begin{aligned}
R_{\gamma \delta}^{k} E_{\delta}^{m} \delta^{-1}\left(E_{\gamma}^{n}\right)=R_{\gamma \delta} E_{\gamma \delta}^{k} E_{\delta}^{m} \delta^{-1}\left(E_{\delta}^{n}\right) & =\left(R_{\delta} E_{\delta}^{m}\right)\left(\delta^{-1}\left(R_{\gamma}\right) \delta^{-1}\left(E_{\gamma}^{n}\right)\right) E_{\gamma \delta}^{k} \\
& =R_{\delta}^{m} \delta^{-1}\left(R_{\gamma}^{n}\right) E_{\gamma \delta}^{k}
\end{aligned}
$$

in 3 for every $k, m, n$, by Lemma 5 (iv) and Lemma 7 (iii). Applying $\gamma \delta$ to both sides, we obtain

$$
\gamma \delta\left(R_{\gamma \delta}^{k}\right) \gamma \delta\left(E_{\delta}^{m}\right) \gamma\left(E_{\gamma}^{n}\right)=\gamma \delta\left(R_{\delta}^{m}\right) \gamma\left(R_{\gamma}^{n}\right) \gamma \delta\left(E_{\gamma \delta}^{k}\right)
$$

and this yields

$$
\begin{aligned}
\left\|\gamma \delta\left(R_{\gamma \delta}^{k} E_{\delta}^{m} \delta^{-1}\left(E_{\gamma}^{n}\right) A\right)-\gamma \delta\left(R_{\gamma \delta}^{r} E_{\delta}^{s} \delta^{-1}\left(E_{\gamma}^{t}\right) A\right)\right\|_{\phi} \\
\quad=\left\|U_{\gamma \delta}\left(E_{\gamma \delta}^{k} E_{\delta}^{m} \delta^{-1}\left(E_{\gamma}^{n}\right) A-E_{\gamma \delta}^{r} E_{\delta}^{s} \delta^{-1}\left(E_{\gamma}^{t}\right) A\right)\right\|_{\phi} \\
\quad=\dot{\phi}\left(\left(E_{\gamma \delta}^{k} E_{\delta}^{m} \delta^{-1}\left(E_{\gamma}^{n}\right)-E_{\gamma \delta}^{r} E_{\delta}^{s} \delta^{-1}\left(E_{\gamma}^{t}\right)\right)^{2} A^{*} A\right)^{1 / 2}
\end{aligned}
$$

From this we see that all of the possible iterated limits of

$$
\left\{\gamma \delta\left(R_{\gamma \delta}^{k} E_{\delta}^{m} \delta^{-1}\left(E_{\gamma}^{n}\right) A\right)\right\}_{k, m, n}
$$

exist and are equal. Indeed, the functional $B \rightarrow \dot{\phi}\left(B A^{*} A\right)$ is $\sigma$-weakly continuous on $\mathfrak{A}$ and the sequences $\left\{E_{\gamma \delta}^{k}\right\},\left\{E_{\delta}^{m}\right\}$ and $\left\{\delta^{-1}\left(E_{\gamma}^{n}\right)\right\}$ converge to 1 strongly in the unit sphere of 3 . But we now have that

$$
\begin{aligned}
U_{\gamma \delta}(A) & =\lim _{m} U_{\gamma \delta}\left(E_{\delta}^{m} A\right)=\lim _{m} \lim _{n} U_{\gamma \delta}\left(E_{\delta}^{m} \delta^{-1}\left(E_{\gamma}^{n}\right) A\right) \\
& =\lim _{m} \lim _{n} \lim _{k} \gamma \delta\left(R_{\gamma \delta}^{k} E_{\delta}^{m} \delta^{-1}\left(E_{\gamma}^{n}\right) A\right) \\
& =\lim _{k} \lim _{m} \lim _{n} \delta\left(R_{\gamma \delta}^{k} E_{\delta}^{m} \delta^{-1}\left(E_{\gamma}^{n}\right) A\right) \\
& =\lim _{k}\left(\lim _{m} \lim _{n} \gamma\left(R_{\gamma}^{n} \delta\left(R_{\delta}^{m} A E_{\gamma \delta}^{k}\right)\right)\right) \\
& =\lim _{k} U_{\gamma} U_{\delta}\left(A E_{\gamma \delta}^{k}\right)=U_{\gamma} U_{\delta} A
\end{aligned}
$$


by relations (3) and (2) and the fact that $\lim _{m} E_{\delta}^{m} A=A, \lim _{n} E_{m}^{\delta} \delta^{-1}\left(E_{\gamma}^{n}\right) A$ $=E_{m}^{\delta} A$, and $\lim _{k} A E_{\gamma \delta}^{k}=A$ in $H$. Therefore, we have that $U_{\gamma \delta}=U_{\gamma} U_{\delta}$ because $\mathrm{m}^{1 / 2}$ is dense in $H$. Finally $U_{\gamma-1}=U_{\gamma}^{-1}$ since $U_{\varepsilon}=1$. This completes the proof that $\gamma \rightarrow U_{\gamma}$ is a homomorphism of $\Gamma$.

III. We now show that $U_{\gamma} A U_{\gamma}^{-1}=\gamma(A)$ for every $A \in \mathfrak{O}$ and $\gamma \in \Gamma$. We recall that each element of $\mathfrak{A}$ has been identified with its image under the canonical isomorphism induced by $\phi$. For every $B \in \mathrm{m}^{1 / 2}$, we have that

$$
\begin{aligned}
U_{\gamma} A U_{\gamma}^{-1} B & =\lim _{n} U_{\gamma}\left(A \gamma^{-1}\left(R_{\gamma-1}^{n} B\right)\right) \\
& =\lim _{n} U_{\gamma}\left(\gamma^{-1}\left(R_{\gamma-1}^{n} \gamma(A) B\right)\right)=\lim _{n} \lim _{m} \gamma\left(R_{\gamma}^{m}\right) R_{\gamma-1}^{n} \gamma(A) B \\
& =\lim _{n} \lim _{m} \gamma\left(E_{\gamma}^{m}\right) E_{\gamma-1}^{n} \gamma(A) B
\end{aligned}
$$

because $\gamma^{-1}\left(R_{\gamma-1}^{n} B\right)$ and $\gamma^{-1}\left(R_{\gamma-1}^{n} \gamma(A) B\right)$ are in $\mathfrak{m}^{1 / 2}$ and because $\gamma\left(R_{\gamma}^{m}\right) R_{\gamma-1}^{n}=\gamma\left(E_{\gamma}^{m}\right) E_{\gamma^{-1}}^{n}$ by relation (1). But we have that

$$
\lim _{n} \lim _{m} \gamma\left(E_{\gamma}^{m}\right) E_{\gamma^{-1}}^{n} \gamma(A) B=\lim _{n} E_{\gamma^{-1}}^{n} \gamma(A) B=\gamma(A) B .
$$

Therefore, we have that $U_{\gamma} A U_{\gamma}^{-1} B=\gamma(A) B$ for every $B \in \mathrm{m}^{1 / 2}$ and consequently $U_{\gamma} A U_{\gamma}^{-1}=\gamma(A)$.

IV. We now prove that $\gamma \rightarrow U_{\gamma}$ is continuous. We first show that $\left(B, U_{\gamma} A\right) \rightarrow(B, A)$ as $\gamma \rightarrow \varepsilon$ for every $A$ and $B$ in $\mathrm{m}^{+}$. Since $B \in \mathrm{m}$ and since $\gamma(A) \rightarrow A$ (strongly) as $\gamma \rightarrow \varepsilon$, we have that $\dot{\phi}(\gamma(A) B) \rightarrow \dot{\phi}(A B)$ as $\gamma \rightarrow \varepsilon$. Thus, it is sufficient to show that $\left\{\left(B, U_{\gamma} A\right)-\dot{\phi}(\gamma(A) B)\right\}$ converges to 0 as $\gamma$ tends to $\varepsilon$. We have that

$$
\left|\left(B, U_{\gamma} A\right)-\dot{\phi}(\gamma(A) B)\right|=\lim _{n}\left|\dot{\phi}\left(\gamma\left(R_{\gamma}^{n} A\right) B\right)-\dot{\phi}\left(\gamma\left(E_{\gamma}^{n} A\right) B\right)\right|
$$

since $\lim _{n} \gamma\left(E_{\gamma}^{n}\right)=1$ (strongly) and $C \rightarrow \dot{\phi}(C B)$ is strongly continuous on $\mathfrak{A}$. The operator $R_{\gamma}^{n}$ is a positive and so there is a positive operator $S_{\gamma}^{n}$ in the unit sphere of $3 E_{\gamma}^{n}$ such that $\left(R_{\gamma}^{n}+E_{\gamma}^{n}\right) S_{\gamma}^{n}=E_{\gamma}^{n}$. On account of the manner in which the $E_{\gamma}^{n}$ are ordered, we may find a positive element $S_{\gamma}$ in the unit sphere of 3 such that $S_{\gamma} E_{\gamma}^{n}=S_{\gamma}^{n}$. We then obtain that

and that

$$
R_{\gamma}^{n}-E_{\gamma}^{n}=\left(R_{\gamma}^{n}-E_{\gamma}^{n}\right)\left(R_{\gamma}^{n}+E_{\gamma}^{n}\right) S_{\gamma}=\left(\left(R_{\gamma}^{n}\right)^{2}-E_{\gamma}^{n}\right) S_{\gamma}
$$

$$
\begin{aligned}
& \dot{\phi}\left(\gamma\left(A\left(R_{\gamma}^{n}-E_{\gamma}^{n}\right)\right) B\right)=\dot{\phi}\left(\gamma\left(A\left(\left(R_{\gamma}^{n}\right)^{2}-E_{\gamma}^{n}\right) S_{\gamma}\right) B\right) \\
& \quad=\dot{\phi}\left(\gamma\left(R_{\gamma}^{n} S_{\gamma} A^{1 / 2}\right) \gamma\left(R_{\gamma}^{n} A^{1 / 2} \gamma^{-1}(B)\right)\right)-\dot{\phi}\left(\gamma\left(A E_{\gamma}^{n} S_{\gamma}\right) B\right) .
\end{aligned}
$$

We notice that $A^{1 / 2} \in \mathrm{m}^{1 / 2}$. Thus, from (4) and (5), we get

$$
\begin{aligned}
\left|\left(B, U_{\gamma} A\right)-\dot{\phi}(\gamma(A) B)\right| & =\left|\left(U_{\gamma}\left(A^{1 / 2} \gamma^{-1}(B)\right), U_{\gamma}\left(S_{\gamma} A^{1 / 2}\right)\right)-\dot{\phi}\left(\gamma\left(A S_{\gamma}\right) B\right)\right| \\
& =\left|\left(A^{1 / 2} \gamma^{-1}(B), S_{\gamma} A^{1 / 2}\right)-\dot{\phi}\left(\gamma\left(A S_{\gamma}\right) B\right)\right| \\
& =\left|\dot{\phi}\left(S_{\gamma} A \gamma^{-1}(B)\right)-\dot{\phi}\left(\gamma\left(A S_{\gamma}\right) B\right)\right|
\end{aligned}
$$


because $U_{\gamma}$ is a unitary operator. We now have the correct estimates and shall complete the proof using an argument by contradiction. Assume there is a $\varrho>0$ and a net $\left\{\gamma_{i}\right\}$ in $\Gamma$ that converges to $\varepsilon$ such that

$$
\left|\left(B, U_{\gamma_{t}} A\right)-\dot{\phi}\left(\gamma_{i}(A) B\right)\right| \geqq \varrho
$$

for every $i$. Because the unit sphere of 3 is weakly compact, we may assume (by passing to a subnet if necessary) that $\left\{S_{\gamma_{1}}\right\}$ converges to an element $S$ in the unit sphere of 3 . By the joint continuity hypothesis (or by Corollary 3 if $\Gamma$ is locally compact) we conclude that $\left\{\gamma_{i}\left(S_{\gamma_{2}}\right)\right\}$ converges weakly to $S$. By the continuity hypothesis, we then can conclude that $\left\{S_{\gamma_{i}} \gamma_{i}^{-1}(B)\right\}$ and $\left\{\gamma_{i}\left(S_{\gamma_{1}}\right) \gamma_{i}(A)\right\}$ converge weakly $S B$ and $S A$ respectively. From (6), we get

$$
\lim _{i}\left|\left(B, U_{\gamma_{i}} A\right)-\dot{\phi}\left(\gamma_{i}(A) B\right)\right|=|\dot{\phi}(S A B)-\dot{\phi}(S A B)|=0 .
$$

This contradicts the choice of $\left\{\gamma_{i}\right\}$. Hence, we have that

$$
\lim _{\gamma \rightarrow \varepsilon}\left(B, U_{\gamma} A\right)=(B, A) .
$$

Since linear combinations of $\mathrm{m}^{+}$are dense in $H$, we have that

$$
\lim _{\gamma \rightarrow \varepsilon}\left(x, U_{\gamma} y\right)=(x, y) \text { for every } x, y \text { in } H .
$$

Because the map $\gamma \rightarrow \gamma \delta$ is continuous at $\varepsilon$ on $\Gamma$ and because $\gamma \rightarrow U_{\gamma}$ is a homomorphism, we see that

$$
\lim _{\gamma \rightarrow \delta}\left(x, U_{\gamma} y\right)=\left(x, U_{\delta} y\right)
$$

for every $x, y$ in $H$. Therefore, the map $y \rightarrow U_{\gamma}$ is continuous. Q.E.D.

\section{Applications to Algebras in Standard Form}

A semi-finite von Neumann algebra $\mathfrak{A}$ on a Hilbert space $H$ is said to be in standard form if there is a Hilbert algebra $\boldsymbol{A}$ that is dense in $H$ such that $\mathfrak{A}$ is generated by the extension to $H$ of the left multiplication operators of $\boldsymbol{A}$. Every semi-finite von Neumann algebra is isomorphic to a von Neumann algebra in standard form [7 and 9; I, §5-6].

The next theorem applies Theorem 8 to algebras in standard form. It is known under more restrictive hypotheses ([14], [16; Remark 4.75], $[18 ; \S 2])$.

Theorem 9. Let $\mathfrak{A}$ be a semi-finite von Neumann algebra in standard form on a Hilbert space $H$. Let $\Gamma$ be a continuous automorphism group of $\mathfrak{A}$. If $\Gamma$ is not locally compact, then assume $\Gamma$ is jointly continuous at the origin on the cartesian product of $\Gamma$ and the unit sphere of the center 3 of $\mathfrak{U}$. Then the automorphism group $\Gamma$ is unitarily implemented on $H$. 
Proof. Let 3 be identified with the space of all essentially bounded measurable functions on the locally compact space $Z$ with respect to a Radon measure $v$. Let $\omega$ be the normal semi-finite trace given by the essential upper integral defined by $v$ on the space $\hat{3}^{+}$of all positive (finite or infinite) measurable functions on $Z$. Let $\Phi$ be a normal semifinite faithful 3 -trace on $\mathfrak{A}^{+}$. This is an extended 3 -module homomorphism on $\mathfrak{A}^{+}$in the sense of Definition 6. The function $\phi=\omega \cdot \Phi$ is a normal semi-finite faithful trace $\mathfrak{A}^{+}$(cf. [9; III, §4]). For every $\gamma \in \Gamma$, we may easily verify that $\phi \cdot \gamma$ is also a normal semi-finite faithful trace on $\mathfrak{A}^{+}$. Hence, there is a normal semi-finite faithful 3 -trace $\Phi_{\gamma}$ on $\mathfrak{A}^{+}$such that $\phi \cdot \gamma=\omega \cdot \Phi_{\gamma}$ (cf. [9; III, $\S 4$, Proposition 3]). However, there is a $Q_{\gamma}$ in $\hat{3}^{+}$such that $Q_{\gamma} \Phi=\Phi_{\gamma}[9 ;$ III, $\S 4$, Theorem 2]. Thus, there is a $Q_{\gamma}$ in $\hat{3}^{+}$such that $\omega \cdot \Phi \cdot \gamma=\omega \cdot\left(Q_{\gamma} \Phi\right)$ for every $\gamma \in \Gamma$. By Theorem 8 , we conclude that there is a unitary representation $U$ of $\Gamma$ on $H_{\phi}$ such that $\pi_{\phi}(\gamma(A))=U_{\gamma} \pi_{\phi}(A) U_{\gamma}^{-1}$. Here $\pi_{\phi}$ is the canonical representation induced by $\phi$ on the Hilbert space $H_{\phi}$. The algebra $\pi_{\phi}(\mathfrak{A})$ on $H_{\phi}$ is also in standard form $[9 ; \mathrm{I}, \S 6$, Theorem 2$]$; and therefore, the isomorphism $\pi_{\phi}$ of $\mathfrak{A}$ onto $\pi_{\phi}(\mathfrak{U})$ is implemented by an isometric isomorphism of $H$ onto $H_{\phi}[9 ; \mathrm{I}, \S 6$, Theorem 4]. This means that $\Gamma$ is also unitarily implemented on $H$. Q.E.D.

The next corollary extends the results of Kallman [18]. It also extends results of Henle [14] in the semi-finite case except that Henle assumed only measurability while we assumed continuity owing to the noncountable situation before us.

Corollary 10. Let $\mathfrak{A}$ be a semi-finite von Neumann algebra with properly infinite commutant on a separable Hilbert space $H$ and let $\Gamma$ be a continuous automorphism group of $\mathfrak{A}$. If $\Gamma$ is not locally compact, then assume that $\Gamma$ is jointly continuous at the origin on the cartesian product of $\Gamma$ and the unit sphere of the center of $\mathfrak{A}$. Then $\Gamma$ is unitarily implemented on $\mathrm{H}$.

Remark. If $\Gamma$ leaves the center of $\mathfrak{A}$ elementwise invariant, we may replace the condition that $H$ is separable by the condition that $\mathfrak{A}$ is countably decomposable. We also note that here no joint continuity hypothesis is needed.

Proof. Let $\phi$ be a normal faithful semi-finite trace on $\mathfrak{A}$, and let $\pi_{\phi}$ be the canonical representation of $\mathfrak{A}$ on $H_{\phi}$ induced by $\phi$. There is a unitary representation $U$ of $\Gamma$ on $H_{\phi}$ such that $\pi_{\phi}(\gamma(A))=U_{\gamma} \pi_{\phi}(A) U_{\gamma}^{-1}$ for every $\gamma \in \Gamma$ (Theorem 8). Let $K$ be a Hilbert space with countably infinite dimension. The commutant of $\dot{\pi}_{\phi}(\mathfrak{A}) \otimes C_{K}$ on the tensor product $H_{\phi} \otimes K$ is $\pi_{\phi}(\mathfrak{U})^{\prime} \otimes L(K)$. Here $C_{K}$ is the algebra of scalar operators on $K$ and $L(K)$ is the algebra of all bounded operators on $K$. But $\pi_{\phi}(\mathfrak{A})$ and $\pi_{\phi}(\mathfrak{U})^{\prime}$ are anti-isomorphic and so $\pi_{\phi}(\mathfrak{L})^{\prime}$ is also $\sigma$-finite [9; III, $\S 1$,

19 Commun math Phys., Vol. 25 
Corollary, Theorem 6]. Thus, the algebra $\pi_{\phi}(\mathfrak{A})^{\prime} \otimes L(K)$ is $\sigma$-finite and properly infinite. This means that the isomorphism $A \rightarrow \pi_{\phi}(A) \otimes 1$ of $\mathfrak{A}$ onto $\pi_{\phi}(\mathfrak{A}) \otimes 1$ is spatial [9; III, $\S 8$, Corollary 8]. But the map $\gamma \rightarrow U_{\gamma} \otimes 1$ is a unitary representation of $\Gamma$ on $H_{\phi} \otimes K$. Therefore $\Gamma$ is unitarily implemented on $H$.

We now derive the second application of Theorem 8 .

Let $\mathfrak{A}$ be a von Neumann algebra on a Hilbert space $H$ with cyclic and separating unit vector $x$. Denote the normal faithful state $\omega_{x}(A)$ $=(A x, x)$ on $\mathfrak{A}$ by $\omega$. Then there exists a one-parameter group $\left\{\sigma_{t} \mid-\infty<t<\infty\right\}$ of automorphisms of $\mathfrak{A}$ associated with $\omega$ such that $\omega \cdot \sigma_{t}=\omega$ for all $t$ and such that for every $A, B$ in $\mathfrak{U}$, there is a function $F(\lambda)$ holomorphic on the strip $0<\operatorname{Im} \lambda<1$ and bounded on $0 \leqq \operatorname{Im} \lambda \leqq 1$ with boundary values $F(t)=\omega\left(\sigma_{t}(A) B\right)$ and $F(t+i)=\omega\left(B \sigma_{t}(A)\right)$ (cf. [21; $\S 13])$. The state $\omega$ is said to satisfy the Kubo-Martin-Schwinger (KMS) boundary conditions for 1 with respect to the one-parameter group $\left\{\sigma_{t}\right\}$, and $\left\{\sigma_{t}\right\}$ is called the modular automorphism group associated with $\omega$ $[21, \S 13]$. As is well-known the group $\left\{\sigma_{t}\right\}$ is unitarily implemented on $H$. In fact, we have that $\left(\Sigma A_{i} x, \Sigma B_{i} x\right)=\left(\Sigma \sigma_{t}\left(A_{i}\right) x, \Sigma \sigma_{t}\left(B_{i}\right) x\right)$ for all $A_{1}, \ldots, A_{n}, B_{1}, \ldots, B_{m}$ in $A$. Hence the map $A x \rightarrow \sigma_{t}(A) x$ can be uniquely extended to a unitary map $U_{t}$ of $H$ such that $\sigma_{t}(A)=U_{t} A U_{t}^{-1}$ for every $A \in \mathfrak{A}$. Also the map $t \rightarrow U_{t}$ is a unitary representation of the reals. For future reference we note that $U_{t} x=x$ for all $t$.

We now consider those automorphisms $\gamma$ of $\mathfrak{A}$ such that $\omega \cdot \gamma=\omega Q_{\gamma}$ for some $Q_{\gamma}$ in $\hat{3}^{+}$, where $\hat{\mathbf{Z}}^{+}$is formed with respect to $\omega$ restricted to the center 3 of $\mathfrak{A}$. Before we set up the precise situation, we characterize those automorphisms $\gamma$ for which $Q_{\gamma}$ exists. These automorphisms leave $\omega$ 3-invariant in the terminology of Guichardet and Kastler [13; Definition 8].

The next proposition generalizes Theorem 1 of [15] to automorphisms that do not leave the center elementwise invariant.

Proposition 11. Let $\mathfrak{A}$ be a von Neumann algebra on a Hilbert space $H$ with center 3. Let $x$ be a cyclic and separating vector for $\mathfrak{A}$ and let $\left\{\sigma_{t}\right\}$ be the modular automorphism group associated with the state $\omega=\omega_{x}$. Let $\gamma$ be an automorphism of $\mathfrak{A}$. Then there exists a positive self-adjoint (perhaps unbounded operator) $C$ affiliated with 3 so that $x$ is in the domain of $C$ and $\omega_{C x}=\omega \cdot \gamma$ if and only if $\gamma$ commutes with $\sigma_{t}$ for every $-\infty<t<\infty$.

Proof. Suppose that $C$ exists. For every $n=1,2, \ldots$, let $E_{n}$ be the spectral projection of $C$ associated with $[0, n]$ and let $C E_{n}=C_{n}$. The monotonely increasing sequence $\left\{E_{n}\right\}$ of projections in 3 has least upper bound 1 .

First we show that $\omega\left(\gamma \cdot \sigma_{t} \cdot \gamma^{-1}(A)\right)=\omega(A)$ for all $A \in \mathfrak{A}$ and $-\infty<t<\infty$. We notice that $\sigma_{t}(B)=B$ for all $B \in 3$ [21; Lemma 15.8]. 
We have that

$$
\begin{aligned}
\omega\left(\gamma \cdot \sigma_{t} \cdot \gamma^{-1}(A)\right) & =\left(\sigma_{t}\left(\gamma^{-1}(A)\right) C x, C x\right) \\
& =\lim _{n}\left(\sigma_{t}\left(\gamma^{-1}(A)\right) C_{n} x, C_{n} x\right) \\
& =\lim _{n}\left(\sigma_{t}\left(\gamma^{-1}(A) C_{n}^{2}\right) x, x\right) \\
& =\lim _{n}\left(\gamma^{-1}(A) C_{n}^{2} x, x\right)=\left(\gamma^{-1}(A) C x, C x\right) \\
& =(A x, x) .
\end{aligned}
$$

Hence, the state $\omega$ is invariant under $\gamma \cdot \sigma_{t} \cdot \gamma^{-1}$ for every $-\infty<t<+\infty$.

Now we show for every $A$ and $B$ in $\mathfrak{A}$, there is a complex-valued function $F(\lambda)$ defined on the strip $0 \leqq \operatorname{Im} \lambda \leqq 1$, that is holomorphic in $0<\operatorname{Im} \lambda<1$ and continuous and bounded on $0 \leqq \operatorname{Im} \lambda \leqq 1$, and that satisfies the KMS boundary conditions

and

$$
F(t)=\left(\gamma \cdot \sigma_{t} \cdot \gamma^{-1}(A) B x, x\right)
$$

$$
F(t+i)=\left(B \gamma \cdot \sigma_{t} \cdot \gamma^{-1}(A) x, x\right)
$$

for all $-\infty<t<+\infty$. Let $A^{\prime}=\gamma^{-1}(A)$ and $B^{\prime}=\gamma^{-1}(B)$. For $n=1,2, \ldots$ the function $F_{n}(\lambda)$ defined on the strip $0 \leqq \operatorname{Im} \lambda \leqq 1$ by

$$
\begin{aligned}
F_{n}(\lambda) & =\left(\Delta^{-i \lambda} B^{\prime} C_{n} x, A^{* *} C_{n} x\right) \text { if } \quad 0 \leqq \operatorname{Im} \lambda \leqq 1 / 2 \\
& =\left(A^{\prime} C_{n} x, \Delta^{1-i \bar{\lambda}} B^{\prime *} C_{n} x\right) \text { if } \quad 1 / 2 \leqq \operatorname{Im} \lambda \leqq 1
\end{aligned}
$$

is holomorphic for $0<\operatorname{Im} \lambda<1$ and continuous and bounded for $0 \leqq \operatorname{Im} \lambda \leqq 1$ [22; Remark, p. 37]. Here $\Delta$ is the modular operator. Also we have that

and

$$
\begin{aligned}
F_{n}(t) & =\left(\sigma_{t}\left(A^{\prime}\right) B^{\prime} C_{n} x, C_{n} x\right) \\
& =\left(\gamma \cdot \sigma_{t} \cdot \gamma^{-1}(A) B \gamma\left(E_{n}\right) x, x\right)
\end{aligned}
$$

$$
\begin{aligned}
F_{n}(t+i) & =\left(B^{\prime} \sigma_{t}\left(A^{\prime}\right) C_{n} x, C_{n} x\right) \\
& =\left(B \gamma \cdot \sigma_{t} \cdot \gamma^{-1}(A) \gamma\left(E_{n}\right) x, x\right),
\end{aligned}
$$

for all real $t$. Comparing the values of $F_{n}$ and $F_{m}$ at $s+i t$ with $s$ and $t$ real and $0 \leqq t \leqq 1 / 2$, we obtain

$$
\begin{aligned}
\left|F_{n}(s+i t)-F_{m}(s+i t)\right| \leqq & \left\|\Delta^{t} B^{\prime}\left(C_{n}-C_{m}\right) x\right\|\left\|A^{\prime *} C_{n} x\right\| \\
& +\left\|\Delta^{t} B^{\prime} C_{m} x\right\|\left\|A^{\prime *}\left(C_{n}-C_{m}\right) x\right\|
\end{aligned}
$$

by [22; Remark, p. 37]. But $\left(1+\Delta^{1 / 2}\right)^{-1}$ is a bounded operator such that $\Delta^{t}\left(1+\Delta^{1 / 2}\right)^{-1}$ is bounded with norm not exceeding 1 for $0 \leqq t \leqq 1 / 2$. 
Hence, we have that

$$
\begin{aligned}
\left\|\Delta^{t} B^{\prime}\left(C_{n}-C_{m}\right) x\right\| & \leqq\left\|\left(1+\Delta^{1 / 2}\right) B^{\prime}\left(C_{n}-C_{m}\right) x\right\| \\
& \leqq\left\|B^{\prime}\left(C_{n}-C_{m}\right) x\right\|+\left\|J \Delta^{1 / 2}\left(B^{\prime}\left(C_{n}-C_{m}\right) x\right)\right\| \\
& \leqq 2\left\|B^{\prime}\right\|\left\|\left(C_{n}-C_{m}\right) x\right\|,
\end{aligned}
$$

and that

$$
\left\|\Delta^{t} B^{\prime} C_{m} x\right\| \leqq 2\left\|B^{\prime}\right\| \operatorname{lub}\left\|C_{n} x\right\|
$$

Here $J$ is the conjugate-linear isometric isomorphism of $H$ such that $J \Delta^{1 / 2} D x=D^{*} x$ for every $D \in \mathfrak{A}$. Since $x$ is in the domain of $C$, we see that $\operatorname{lub}\left\|C_{n} x\right\|<+\infty$ and that $\lim _{m, n}\left\|\left(C_{n}-C_{m}\right) x\right\|=0$. Hence $\left\{F_{n}(\lambda)\right\}$ is uniformly Cauchy on $0 \leqq \operatorname{Im} \lambda \leqq 1 / 2$. Likewise we obtain that $\left\{F_{n}(\lambda)\right\}$ is uniformly Cauchy on $1 / 2 \leqq \operatorname{Im} \lambda \leqq 1$. This means that $\left\{F_{n}\right\}$ converges uniformly to a function $F$ on $0 \leqq \operatorname{Im} \lambda \leqq 1$ and hence that $F$ is holomorphic on $0<\operatorname{Im} \lambda<1$ and continuous and bounded on $0 \leqq \operatorname{Im} \lambda \leqq 1$. Now we calculate $F(t)$ and $F(t+i)$ for all real $t$. Since $\left\{\gamma\left(E_{n}\right)\right\}$ converges strongly to 1 , we have that

$$
\begin{aligned}
F(t)=\lim _{n} F_{n}(t) & =\lim _{n}\left(\gamma \cdot \sigma_{t} \cdot \gamma^{-1}(A) B \gamma\left(E_{n}\right) x, x\right) \\
& =\left(\gamma \cdot \sigma_{t} \cdot \gamma^{-1}(A) B x, x\right)
\end{aligned}
$$

and likewise that

$$
F(t+i)=\left(B \gamma \cdot \sigma_{t} \cdot \gamma^{-1}(A) x, x\right),
$$

for all $-\infty<t<+\infty$. This means that $t \rightarrow \gamma \cdot \sigma_{t} \cdot \gamma^{-1}$ is the modular automorphism group of the normal faithful state $\omega$ of $\mathfrak{A}$. By the uniqueness of the modular automorphism group of $\omega$, [21; Theorem 13.2] we obtain that $\gamma \cdot \sigma_{t} \cdot \gamma^{-1}=\sigma_{t}$ for all $-\infty<t<+\infty$.

Conversely, suppose that $\sigma_{t} \cdot \gamma=\gamma \cdot \sigma_{t}$ for every $-\infty<t<\infty$. However, the one-parameter group $\left\{\gamma^{-1} \cdot \sigma_{t} \cdot \gamma\right\}$ of automorphisms is the modular automorphism group of $\omega \cdot \gamma$ [15; Lemma 1], i.e. the normal functional $\omega \cdot \gamma$ satisfies the KMS boundary conditions with respect to $\sigma_{t}$. This means that there is a positive self-adjoint (perhaps unbounded) operator $C$ affiliated with 3 such that the domain of $C$ contains $x$ and that $\omega_{C x}=\omega \cdot \gamma[21$; Theorem 15.4]. Q.E.D.

Let $\mathfrak{U}$ be a von Neumann algebra with center 3 on the Hilbert space $H$ and let $x \in H$ be a cyclic and separating vector for $\mathfrak{A}$. Let $v$ be the measure on the spectrum $Z$ of 3 induced by the relation

$$
(A x, x)=\int_{Z} A^{\wedge}(\zeta) d v(\zeta) .
$$

Here $A^{\wedge}$ is the Gelfand transform of $A \in \mathcal{3}$. Then 3 with its $\sigma$-weak topology is identified with the space $L^{\infty}(Z, v)$ of essentially bounded 
measurable functions on $Z$ with its $\sigma$-weak topology by the Gelfand transform $A \rightarrow A^{\wedge}$. Indeed, the measure $v$ is a so-called perfect measure. Now the projection $E$ of $H$ on the subspace closure $\{A x \mid A \in 3\}$ is an abelian projection of the commutant $3^{\prime}$ of 3 . Since $x$ is a cyclic vector for $3^{\prime}$, the projection $E$ has central support 1 in $3^{\prime}$. Hence, for each $A$ in $3^{\prime}$, there is a unique $\Phi^{\prime}(A)$ in 3 such that $E A E=\Phi^{\prime}(A) E$. The $\operatorname{map} \Phi^{\prime}(A)$ is a positive $\sigma$-weakly continuous 3-module homomorphism of $3^{\prime}$ into 3 . The restriction $\Phi$ of $\Phi^{\prime}$ to $\mathfrak{A}$ is also a positive $\sigma$-weakly continuous 3 -module homomorphism of $\mathfrak{A}$ into 3 . It is also faithful since $\Phi\left(A^{*} A\right)=0$ implies $\left(\Phi\left(A^{*} A\right) x, x\right)=\left(\Phi^{\prime}\left(A^{*} A\right) E x, x\right)=(A x, A x)=0$ and hence that $A=0$.

Proposition 12. Let $\mathfrak{U}$ be a von Neumann algebra on a Hilbert space $H$. Let $x$ be a cyclic and separating vector for $\mathfrak{H}$, and let $\left\{\sigma_{t}\right\}$ be the modular automorphism group of the normal faithful functional $\omega_{x}$. Let $\Gamma$ be a continuous automorphism group of $\mathfrak{A}$ such that $\gamma \cdot \sigma_{t}=\sigma_{t} \cdot \gamma$ for every real $t$. If $\Gamma$ is not locally compact, then assume that $\Gamma$ is jointly continuous at the origin on the cartesian product of $\Gamma$ with the unit sphere of the center of $\mathfrak{A}$. Then the automorphism group $\Gamma$ is uniformly implemented by a unitary representation $U$ of $\Gamma$ on $H$ so that, given any unitary implementation $V$ of $\left\{\sigma_{t}\right\}$ on $H$ such that $V_{t} x=x$, the unitary operators $U_{\gamma}$ and $V_{t}$ commute for every $\gamma \in \Gamma$ and $-\infty<t<\infty$. Furthermore, if $J \Delta^{1 / 2}$ is the polar decomposition of the closure $S$ of the conjugate linear operator $A x \rightarrow A^{*} x(A \in \mathfrak{A})$, then $U_{\gamma}$ commutes with $J$ and $\Delta$ for every $\gamma \in \Gamma$.

Remark 1. As we have seen, such an implementation $V$ of $\left\{\sigma_{t}\right\}$ exists.

Remark 2. In view of Proposition 11, the last statement of Proposition 12 is a generalization of a result of Størmer [20; Lemma 2].

Proof. We preserve the notation of the paragraph introducing this proposition. For every $\gamma \in \Gamma$, there is a positive self-adjoint $C_{\gamma}$ affiliated with the center 3 of $\mathfrak{A}$ such that $x$ is in the domain of $C_{\gamma}$ and such that $\omega_{C_{\gamma} x}=\omega_{x} \cdot \gamma$ (cf. Proposition 11). Let $F_{\gamma}^{n}$ be the spectral projection of $C_{\gamma}$ corresponding to the interval $[0, n]$ and let $C_{\gamma}^{n}=C_{\gamma} F_{\gamma}^{n}$. For every $A \in \mathfrak{U}^{+}$, we have that

$$
\begin{aligned}
\omega_{x} \cdot \gamma(A) & =\left(A C_{\gamma} x, C_{\gamma} x\right) \\
& =\operatorname{lub}_{n}\left(A C_{\gamma}^{n} x, C_{\gamma}^{n} x\right)=\operatorname{lub}_{n}\left(A\left(C_{\gamma}^{n}\right)^{2} x, x\right) \\
& =\operatorname{lub}_{n}\left(\Phi\left(A\left(C_{\gamma}^{n}\right)^{2}\right) x, x\right)=\operatorname{lub}_{n}\left(\left(C_{\gamma}^{n}\right)^{2} \Phi(A) x, x\right)=\omega\left(Q_{\gamma} \Phi(A)\right),
\end{aligned}
$$

where $Q_{\gamma}$ is the least upper bound of the monotonely increasing net $\left\{\left(C_{\gamma}^{n}\right)^{2}\right\}$ in $\hat{3}^{+}$. Thus, the hypotheses of Theorem 8 are satisfied. Let us preserve the notation of this theorem. Let $\pi_{\phi}$ be the canonical homomorphism of $\mathfrak{U}$ induced by $\phi$ on $H_{\phi}$. For $A_{1}, \ldots, A_{n}, B_{1}, \ldots, B_{n}$ in $\mathfrak{A}$, we have $\left(\sum A_{i} x, \Sigma B_{i} x\right)=\phi\left(\left(\Sigma B_{i}\right)^{*}\left(\Sigma A_{i}\right)\right)$. Hence, the space $H$ is spatially 
isomorphic to $H_{\phi}$ under the map $A x \rightarrow A$ of $\mathscr{A} x$ onto $m^{1 / 2}=\mathfrak{A}$. Hence, we may transfer the implementing unitary representation of $\Gamma$ on $H_{\phi}$ to a unitary representation $U$ on $H$ by setting $U_{\gamma} A x=\lim _{n} \gamma\left(R_{y}^{n} A\right) x$ for every $A \in \mathfrak{U}$.

For every $A \in \mathfrak{U}, \gamma \in \Gamma$, and $-\infty<t<+\infty$, we have that

$$
\begin{aligned}
V_{t} U_{\gamma} A x & =\lim _{n} V_{t} \gamma\left(R_{\gamma}^{n} A\right) x \\
& =\lim _{n} \sigma_{t} \cdot \gamma\left(R_{\gamma}^{n} A\right) V_{t} x=\lim _{n} \gamma\left(R_{\gamma}^{n} \sigma_{t}(A)\right) x \\
& =U_{\gamma}\left(\sigma_{t}(A) x\right)=U_{\gamma} V_{t} A x
\end{aligned}
$$

since $V_{t} x=x$ and since $\sigma_{t}(C)=C$ for all $C \in 3$ [21; Lemma 15.8]. Because $\mathfrak{A} x$ is dense in $H$, we get that $V_{t} U_{\gamma}=V_{t} U_{\gamma}$ for all $\gamma, t$.

We now consider the last statement. For every $A \in \mathfrak{A}$ and $\gamma \in \Gamma$, we have that

$$
A x=\lim \gamma\left(R_{\gamma}^{n} A\right) x .
$$

Since $R_{\gamma}^{n}$ is in $3^{+}$, we have that

$$
\lim S \gamma\left(R_{\gamma}^{n} A\right) x=\lim \gamma\left(R_{\gamma}^{n}\right) S(\gamma(A) x)=\lim \gamma\left(R_{\gamma}^{n} A^{*}\right) x=U_{\gamma} A^{*} x .
$$

Using the fact that $S$ is a closed conjugate linear operator, we have that $U_{\gamma} A x$ is in the domain $D(S)$ of $S$ and that $U_{\gamma}^{-1} S U_{\gamma} A x=A^{*} x=S A x$. Now let $y \in \mathfrak{D}(S)$. There is a sequence $\left\{A_{n}\right\}$ in $\mathfrak{A}$ with $\lim A_{n} x=y$ and $\lim A_{n}^{*} x=S y$. However, we have that $\lim U_{\gamma} A_{n} x=U_{\gamma} y$ and $\lim S U_{\gamma} A_{n} x$ $=U_{\gamma} S y$. Since $S$ is closed, we have that $U_{\gamma} y \in \mathfrak{D}(S)$ and that $U_{\gamma}^{-1} S U_{\gamma} y=S y$. This means that $S \subset U_{\gamma}^{-1} S U_{\gamma}$. Since $\gamma$ is arbitrary, we may conclude that $S=U_{y}^{-1} S U_{y}$ for all $\gamma \in \Gamma$. Hence, we have that $U_{y}^{-1} J U_{y}=J$ and $U_{\gamma}^{-1} \Delta^{1 / 2} U_{\gamma}=\Delta^{1 / 2}$ (cf. [11; XII.7.6] for the analogous result for closed linear operators). Finally, we get that $U_{\gamma}^{-1} \Delta U_{\gamma}=\Delta$. Q.E.D.

Added in Proof on February 10, 1972. A more general form of Proposition 11 was obtained independently by F. Combes [Proposition 4.17, Compositio Math. 23, 49-77 (1971)].

\section{References}

1. Aarnes, J.: On the continuity of automorphic representations of groups. Commun. math. Phys. 7, 332-336 (1968).

2. - Continuity of group representations, with applications to $C^{*}$-algebras. J. Functional Analysis 5, 14-36 (1970).

3. Borchers, H. J.: On the implementability of automorphism groups. Commun. math. Phys. 14, 305-314 (1969).

4. Bourbaki, N.: Integration, Chapters I-V, Act. Sci. Ind. no. 1175, 1244. Paris: Hermann 1965, 1967.

5. Busby, R., Smith,H.: Representations of twisted group algebras. Trans. Am. Math. Soc. 149, 503-537 (1970). 
6. Dixmier, J.: Applications $\square$ dans les anneaux d'opérateurs. Compositio Math. 10, 1 - 55 (1952).

7. - Algèbres quasi-unitaires. Comment. Math. Helv. 26, 275-322 (1952).

8. - Les $C^{*}$-algèbres et leurs représentations. Paris: Gauthier-Villars 1964.

9. - Les algèbres d'opérateurs dans l'éspace Hilbertien. Paris: Gauthier-Villars 1969.

10. Doplicher,S., Kastler, D., Robinson, D.: Covariance algebras in field theory and statistical mechanics. Commun. math. Phys. 3, 1-28 (1966).

11. Dunford, N., Schwartz, J.: Linear operators, Parts I and II. New York: Interscience 1957, 1963.

12. Fell,J.M.G.: An extension of Mackey's method to Banach *-algebraic bundles. Mem. Am. Math. Soc. no. 90, 1-168 (1969).

13. Guichardet, A., Kastler, D.: Désintégration des états quasi-invariants des $C^{*}$-algèbres. J. Math. Pures Appl. 49, 349 - 380 (1970).

14. Henle, M.: Spatial representation of groups of automorphisms of von Neumann algebras with properly infinite commutants. Commun. math. Phys. 19, 273-275 (1970).

15. Herman, R., Takesaki, M.: States and automorphism groups of operator algebras. Commun. math. Phys. 19, 142-160 (1970).

16. Kadison, R. V.: Transformation of states in operator theory and dynamics. Topology 3 (Suppl.) 2, 177-198 (1965).

17. Kallman, R. R.: A remark on a paper by J. F. Aarnes. Commun. math. Phys. 14, 13-14 (1969).

18. - Spatially mduced groups of automorphisms of certain von Neumann algebras. Trans. Am. Math. Soc. 156, 505-516 (1971).

19. Sirugue. M., Winnink, M.: Constraints imposed upon a state of a system that satısfies the K.M.S. boundary condition. Commun. math. Phys. 19, 161-168 (1970).

20. Størmer, B.: Automorphisms and invariant states of operator algebras. Acta Math 127, $1-10$ (1971).

21. Takesaki, M.: Tomita's theory of modular Hilbert algebras and its applications. Berlin, Heidelberg, New York: Springer 1970.

22. - Disjointness of the KMS-states of different temperatures. Commun. math. Phys. 17, $33-41(1970)$.

23. Tomita, M.: Standard forms of von Neumann algebras, the Vth Functional Analysis Symposium of Math. Soc. of Japan, Sendai, 1967.

\author{
H. Halpern \\ Department of Mathematics \\ University of Cincinnati \\ Cincinnati, Ohio 45221, USA
}

